\title{
Status of the Storage Ring Design at FAIR
}

\author{
S. Litvinov*, C. Dimopoulou, A. Dolinskii, O. Gorda, F. Nolden, M. Steck \\ E-mail:S.Litvinovegsi.de \\ GSI Helmholtzzentrum für Schwerionenforschung GmbH, 64291, Darmstadt, Germany
}

\begin{abstract}
The large acceptance Collector Ring (CR) together with the High Energy Storage Ring (HESR) is the storage ring which will be realized in the Modularized Start Version (MSV) of the FAIR project. It will be operated in three ion-optical modes, two of them providing fast pre-cooling of either antiprotons or RIBs. The CR design was recently adapted to the use as a pre-cooling ring for subsequent beam accumulation in the HESR. The third mode, namely the isochronous mode, is a special ion-optical setting for mass measurement of exotic very short-lived nuclei. In this mode the CR will be operated as a Time-Of-Flight (TOF) spectrometer. The latest results of the general storage ring concept at FAIR will be presented and future perspectives of the ring complex development will be discussed.
\end{abstract}

8th International Conference on Nuclear Physics at Storage Rings-Stori11,

October 9-14, 2011

Laboratori Nazionale di Frascati, Italy

${ }^{*}$ Speaker. 


\section{Introduction}

Acceleration of high intensity primary beams and their conversion to secondary beams is the main mission of the proposed Facility for Antiproton and Ion Research (FAIR) [1]. The existing GSI accelerator system with the UNILAC linear accelerator and the heavy ion synchrotron SIS18 will serve as injector complex for the new synchrotron SIS100. Two production targets, one for antiproton production using a primary proton beam and one for rare isotope production by fragmentation or fission of heavy ions, in combination with subsequent magnetic separators, will provide the secondary beams. In a complex of storage rings the secondary beams will be prepared for the users. Beam cooling will be crucial to prepare high quality secondary beams. Stochastic cooling will provide pre-cooling of the hot secondary beams, for antiprotons it is also employed in beam accumulation.

The large acceptance Collector Ring (CR) together with the High Energy Storage Ring (HESR) are the storage rings which will be realized in the Modularized Start Version (MSV) of the FAIR project. The overview of the FAIR facility is presented in Fig. 1.

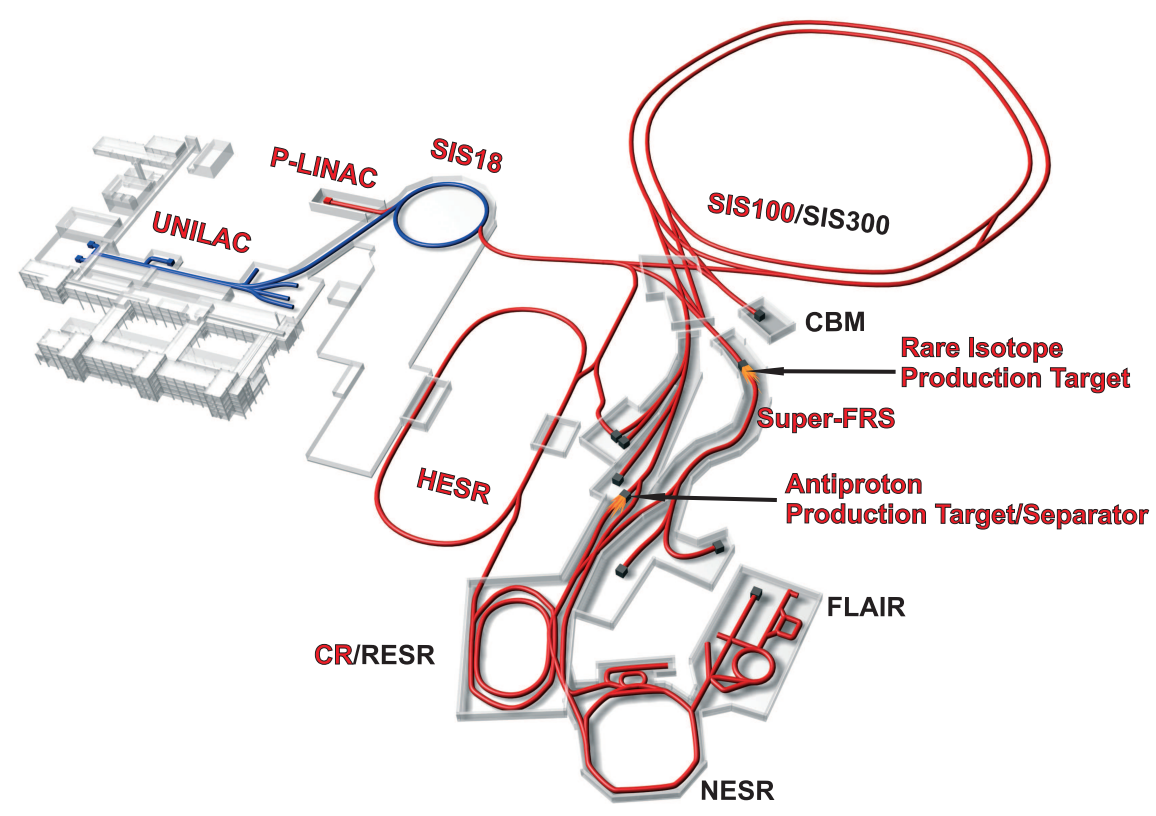

Figure 1: Layout of the FAIR facility. The components which will be realized in the Modularized Start Version of the FAIR are marked in red.

The purpose of the CR is the collection and pre-cooling of antiprotons (for the subsequent antiproton transport into the HESR) and Rare Isotope Beams (RIBs). Additionally the CR will be operated in the isochronous mode for mass measurements of RIBs.

The HESR with a circumference of $575 \mathrm{~m}$ and a magnetic rigidity of $50 \mathrm{Tm}$ is designed for the accumulation and storage of antiprotons. The antiprotons will be injected at $3 \mathrm{GeV}$ and then be prepared according to the requirements of the experiment. The energy ranges from a maximum of 14.1 GeV down to a minimum of $0.83 \mathrm{GeV}$. The antiprotons are accelerated or decelerated in the HESR to the required energy with a ramp rate of $0.025 \mathrm{~T} / \mathrm{s}$. More detailed information one can find in Refs. [2, 3]. 


\section{The Collector Ring (CR)}

The Collector Ring (CR) offers large acceptances, both transversely and longitudinally, which is important for the efficient use of the secondary beams injected from the production targets and separators. Its design is governed by the stochastic cooling system which requires proper mixing conditions and beta functions [4]. Since the accumulator ring RESR is excluded from the start version of FAIR, the CR has been adopted to the use as a pre-cooling ring for direct subsequent beam accumulation in the HESR. Antiproton and Rare Isotope Beams (RIBs), are injected at the maximum magnetic rigidity of $13 \mathrm{Tm}$. The velocities of the two species differ significantly, the relativistic velocity is $\beta=0.83$ for ions and $\beta=0.97$ for antiprotons. Therefore, in order to provide the best conditions for the stochastic cooling system for both beam species two different ion-optical settings have been calculated. Dispersion free straight sections for injection and extraction and for the installation of the rf system are another feature of the lattice. The circumference of the CR is $221.5 \mathrm{~m}$. In the antiproton operation mode the momentum acceptance is $\Delta p / p= \pm 3 \%$ and the transverse acceptance is $240 \mathrm{~mm}$ mrad in both planes. In the RIB mode the momentum acceptance is $\Delta p / p= \pm 1.5 \%$ and the transverse acceptance is $200 \mathrm{~mm} \mathrm{mrad}$, correspondingly. in both planes. The momentum slip factor is rather small for both optical modes $(\eta=-0.011$ for antiprotons, $\eta=0.186$, for RIBs). The CR is schematically illustrated in Fig. 2.

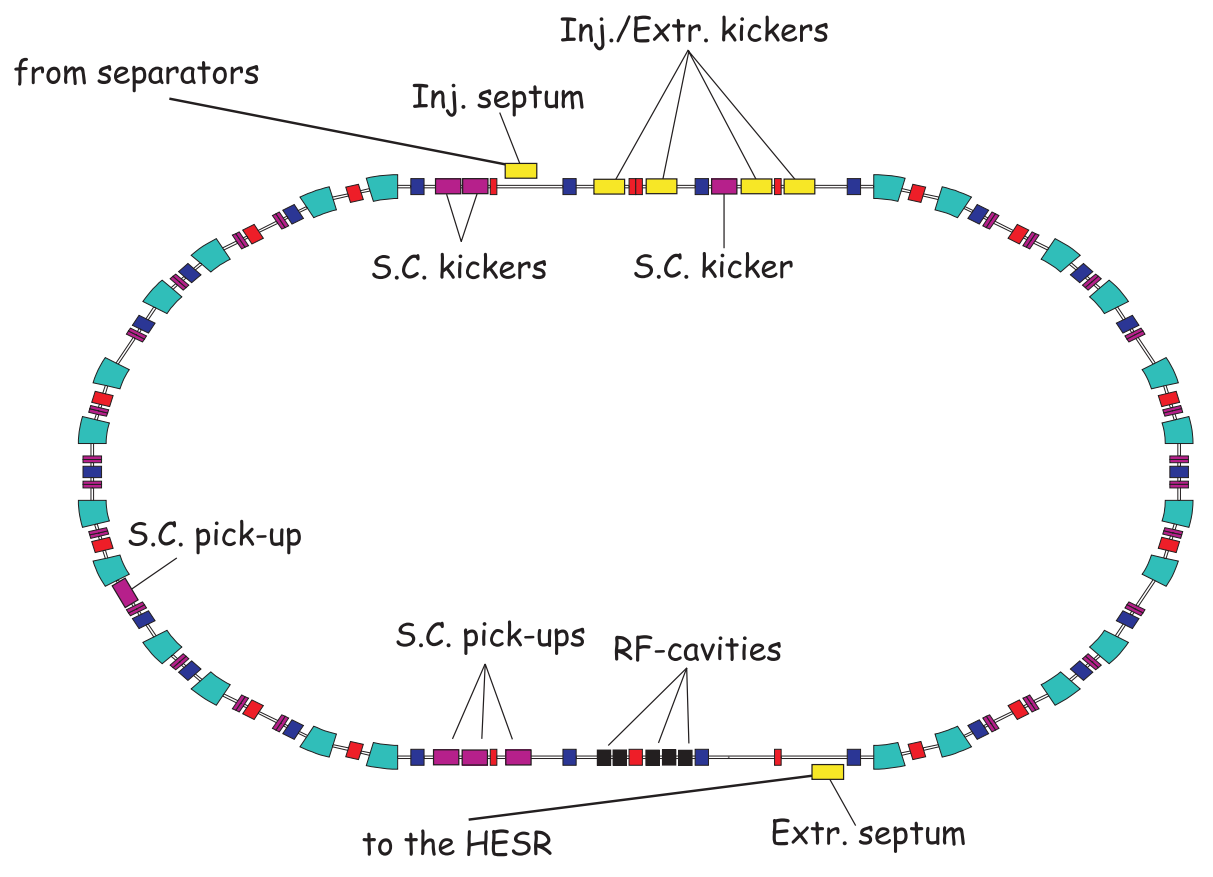

Figure 2: Layout of the Collector Ring (CR).

\subsection{Fast Beam Rotation}

Before stochastic cooling is applied a bunch rotation and debunching system reduces the momentum spread of the short bunch of secondary particles from $\pm 3 \%$ to $\pm 0.7 \%$. This results in better initial conditions for stochastic cooling and in a shorter total cooling time. The rf system of the CR [5] is designed for high voltage gradient during bunch rotation, but it acts only over some 
hundred turns on the beam. Although each $1 \mathrm{~m}$ long cavity has to provide a voltage of $40 \mathrm{kV}$, the average power dissipation will not exceed $2 \mathrm{~kW}$. The use of magnetic alloy material is foreseen in order to achieve the high electric field strength. Due to the small average power dissipation forced air cooling of the magnetic alloy rings will be sufficient. No change of the beam energy with the rf system is foreseen in the $\mathrm{CR}$, therefore the frequency range for the operation at harmonic number $\mathrm{h}=1$ from 1.13 to $1.32 \mathrm{MHz}$ is only determined by the difference in velocity of ions and antiprotons when injected at their specific energies. The rotation time is about $1000 \mu \mathrm{s}$ and $600 \mu \mathrm{s}$ for antiprotons and RIBs, correspondingly.

\subsection{Stochastic Cooling}

The stochastic cooling of the CR will be applied to beams with significantly different velocity [6]. The intensities, particularly of rare isotope beams, can vary from very low intensity (a few ions) up to some $10^{8}$. The goal for low ion intensities is a total cooling time of $1.5 \mathrm{~s}$ in order to make full use of the fast cycle of SIS100. The cooling time for antiprotons should be $10 \mathrm{~s}$ at most, $5 \mathrm{~s}$ total cooling time will result in doubling the antiproton production rate. For cost reasons the bandwidth of the cooling system is presently limited to the range 1 to $2 \mathrm{GHz}$, an optional extension to $1-4 \mathrm{GHz}$ will reduce the cooling time.

For the cooling of the low intensity ion beams and for the antiproton cooling high sensitivity of the pick-ups is indispensable. A new slot line structure for the band $1-2 \mathrm{GHz}$ coupled to a micro-strip circuit has been developed [7]. Cooling of the pick-ups to $20 \mathrm{~K}$ with a cold head is included in the design of the vacuum tank. A prototype tank is presently assembled in order to test the concept. Pick-ups and kickers will be moved during the cooling process synchronously with the decreasing emittance of the beam in order to have best signal to noise ratio. Switchable delays allow matching the traveling time of the correction signal to the time of flight of the particles from pick-up to kicker. The designed system has been proven to provide good amplitude and phase flatness in the band from 1 to $2 \mathrm{GHz}$.

The antiprotons will be stored for about $10 \mathrm{~s}$ and rare isotopes for a few seconds. The required beam lifetime for these high energy secondary beams can be achieved in a metal sealed ultrahigh vacuum system without baking.

\subsection{Isochronous Optics}

The third ion-optical mode of the CR is the isochronous optics. This is a special ion-optical setting in which the revolution frequency of circulating ions of one species does not depend on their velocity spread. In this mode the CR will be operated as a time-of-flight spectrometer for shortlived exotic nuclei $\left(\mathrm{T}_{1 / 2}>20 \mu \mathrm{s}\right)$ produced and selected in flight with the Super-FRS fragment separator [8]. This technique for mass measurements has been developed at the ESR at GSI [9]. An advantage of this method is that a large number of nuclei can be measured in one experimental run.

The mass-to-charge ratio $m / q$ of the stored ions circulating in the ring can be measured from the revolution frequency $(f)$ (or revolution time) and the velocity $(v)$ of the ions.

$$
\frac{\Delta f}{f}=-\frac{1}{\gamma_{t}^{2}} \cdot \frac{\Delta(m / q)}{(m / q)}+\left(1-\frac{\gamma^{2}}{\gamma_{t}^{2}}\right) \frac{\Delta v}{v}
$$


where $\gamma$ is the relativistic Lorentz factor and $\gamma_{t}$ the transition point of the ring. The isochronous condition is reached when

$$
\gamma=\gamma_{t}
$$

The $\gamma_{t}$ can be adjusted to the desired $\gamma$ value by a special quadrupole setting. The first isochronous settings have been calculated to be at the transition energy of 1.84 with transverse acceptances of $100 \mathrm{~mm} \mathrm{mrad}$ in both planes and momentum acceptance of $1 \%[10,11,12]$. However, this $\gamma_{t}$ allows the measurements of mass-to-charge ratios only up to $m / q=2.71$ at the maximum $B \rho_{\max }=13$ $\mathrm{Tm}$ [13]. In order to expand the accessible mass region to very exotic neutron-rich nuclei, it is nec-

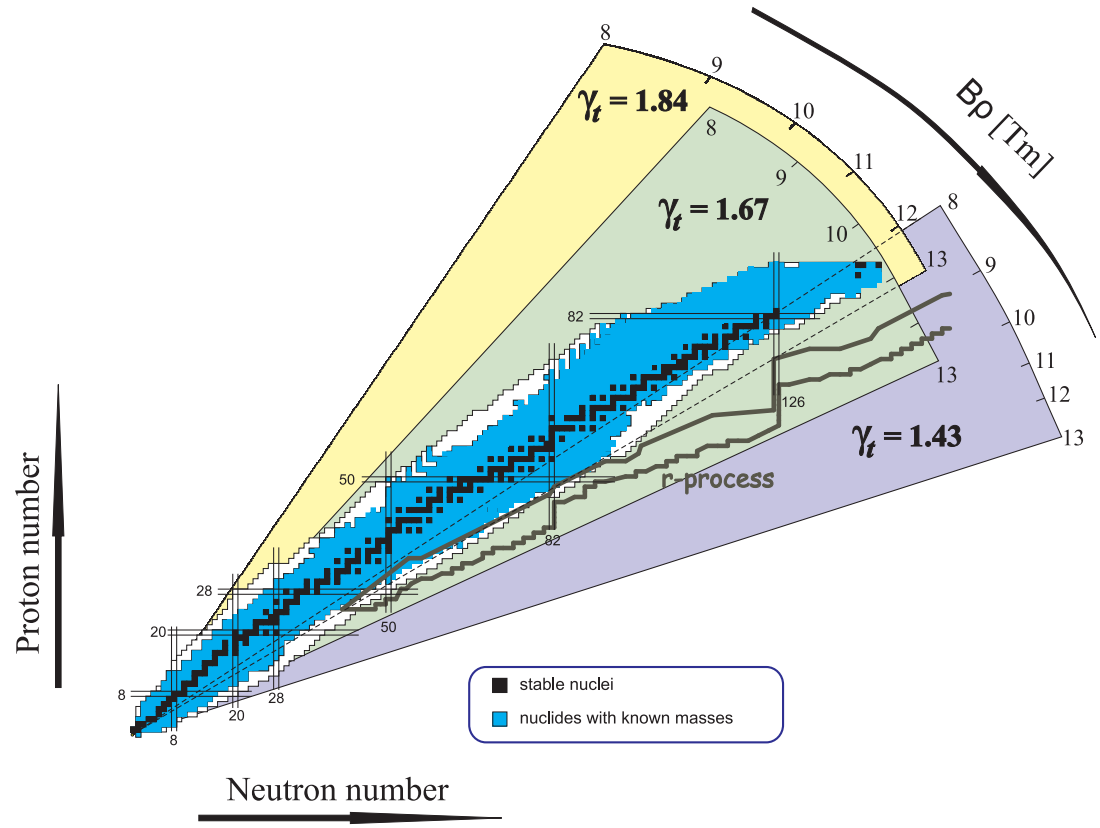

Figure 3: The chart of nuclides. Experimentally known nuclides are shown as blue squares at the corresponding proton and neutron numbers. The stable masses are shown in black color. A predicted astrophysical r-process is shown as a brown curve. Different sectors indicate the region of nuclei which can be accessed with different $\gamma_{t}$ settings and corresponding $B \rho$ of the $\mathrm{CR}$.

essary to reduce the transition energy of the ring further. However, the latter leads to an increase of the dispersion function and correspondingly reduces the longitudinal momentum acceptance. In addition, the mass analysis becomes very complicated due to a small number of reliable reference masses. If at lower energies, the less-exotic isobars in different atomic charge states are stored in the ring simultaneously with the nuclides of interest, at our high energies $(\gamma=1.84)$ the exotic nuclei are produced mainly as fully-ionized atoms and no (less-exotic) isobars are present. Recently, three isochronous settings with different $\gamma_{t}=1.43,1.67,1.84$ have been calculated in detail. They have been calculated in such a way, that the injection scheme is the same for all three cases. With these optics one can cover a wide region of exotic nuclei (see Fig. 3). One can see, that most of neutron-rich nuclides can be accessed with $\gamma_{t}=1.67$ setting, and most exotic light nuclei can be addressed only with the extreme setting corresponding to $\gamma_{t}=1.43$. The transverse acceptance in all three setting is $100 \mathrm{~mm}$ mrad in both planes. The longitudinal acceptance is smallest for $\gamma_{t}=1.43$ mode due to the largest dispersion and corresponds to $\Delta p / p= \pm 0.2 \%$. For the $\gamma_{t}=1.67$ 
and $\gamma_{t}=1.84$ settings the momentum acceptance is $\Delta p / p= \pm 0.4 \%$ and $\Delta p / p= \pm 0.6 \%$ correspondingly. The lattice functions of the three settings are illustrated in Fig. 4.

After first-order calculation of the isochronous setting, one has to correct second-order isochronicity. It can usually be performed with one family of sextupole magnets installed in the dispersive part of the ring. After this correction, the effect of the transverse motion on the revolution time becomes visible. The influence of this effect has been investigated in Ref. [14]. There, it has been pointed out, in order to reach a mass resolving power in the $\mathrm{CR}$ of $10^{5}$ the transverse beam emittance has to be restricted to $10 \mathrm{~mm}$ mrad in both planes. However, that research has been done in first-order approximations. Recent investigations show, that an influence of the transverse emittance on the revolution time can be significantly reduced by a chromaticity correction with two families of sextupole magnets. A dedicated Monte-Carlo simulation has been performed. In the calculations we have used a beam of $10^{5}$ particles with $100 \mathrm{~mm}$ mrad transverse emittance in both planes circulating 100 turns in the CR. The second-order isochronicity was corrected by default. A dependence of

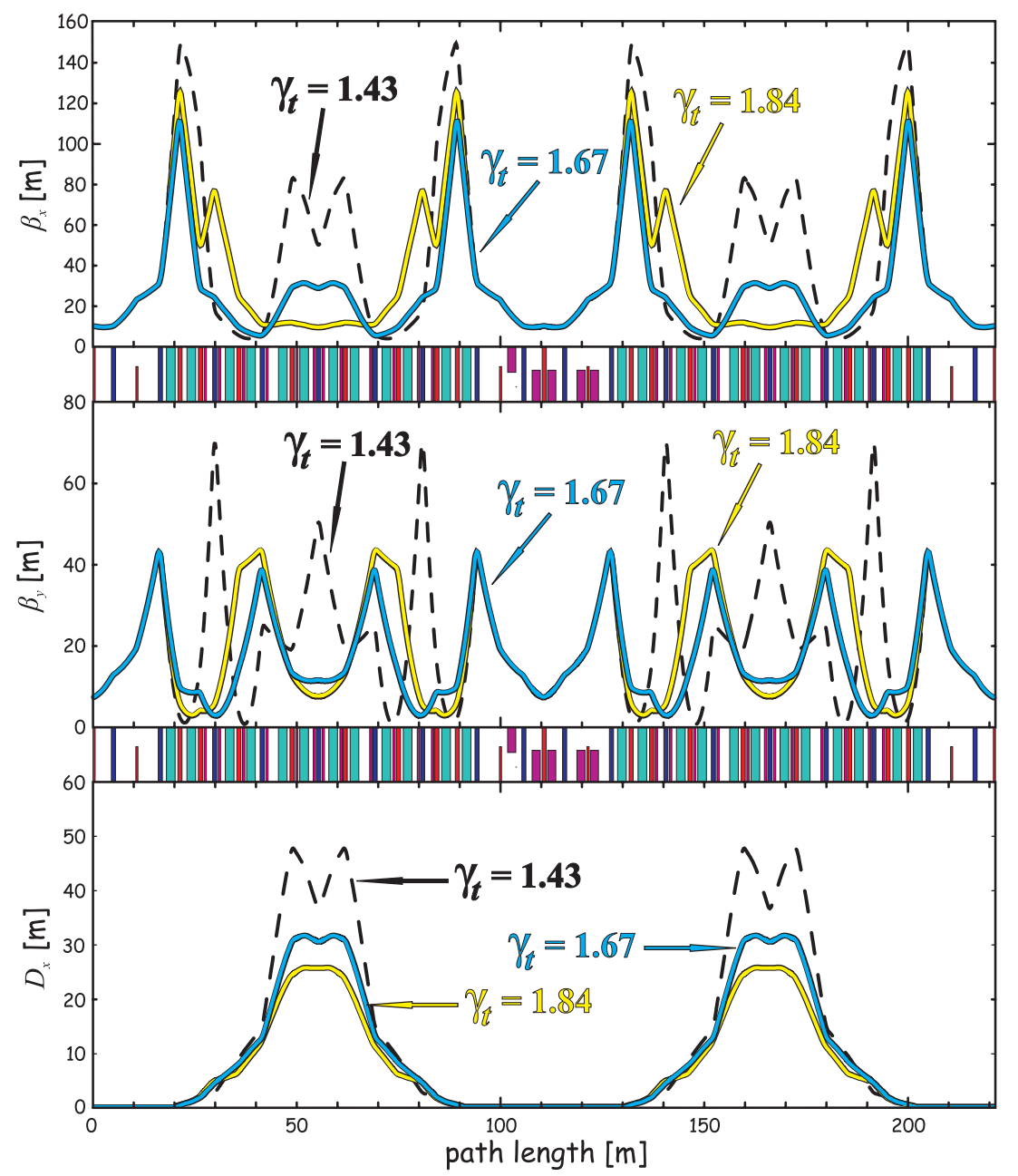

Figure 4: The $\beta_{x}$ - (upper picture), $\beta_{y}$ - (middle picture) and dispersion functions (bottom picture) as a function of the path length calculated for three $\gamma_{t}$ settings. The dashed black, solid blue and solid yellow curves correspond to the $\gamma_{t}=1.43, \gamma_{t}=1.67$ and $\gamma_{t}=1.84$ modes correspondingly. 

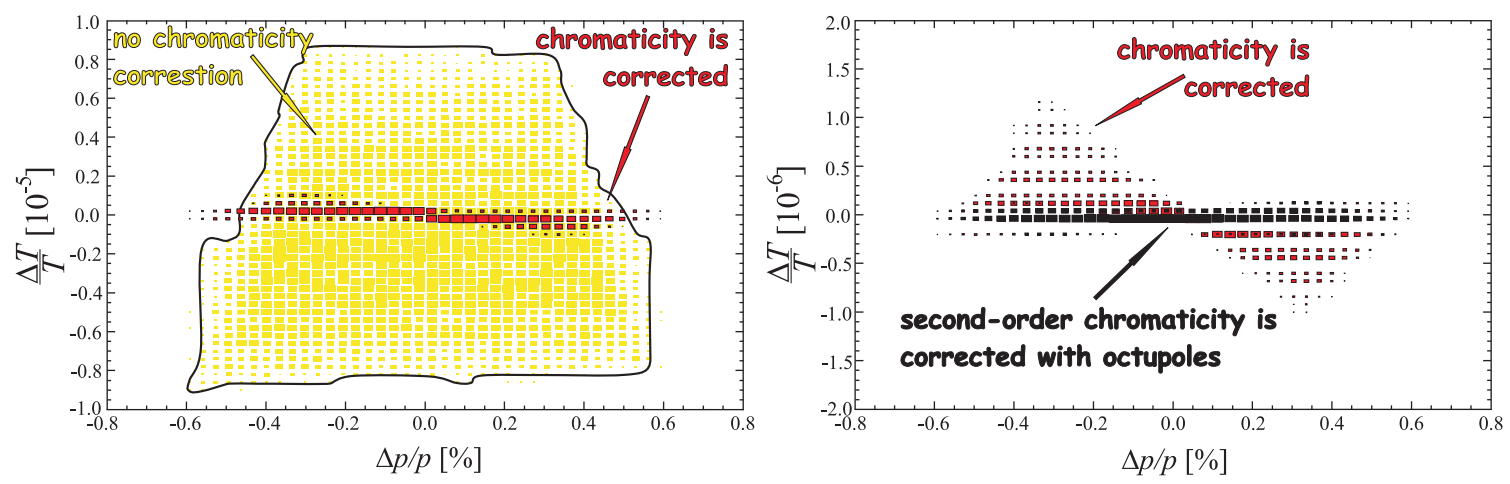

Figure 5: The revolution time as a function of the momentum deviation. Only second-order isochronicity is corrected with one family of sextupole magnets (yellow distribution). Second-order isochronicity and the chromaticity are corrected with three families of sextupole magnets (red distribution). Third-order isochronicity and second-order chromaticity are corrected with three families of octupole magnets (black distribution).

the revolution time on the momentum deviation is presented in Fig. 5. The broad time-of-flight distribution on the left picture (yellow area in Fig. 5) is governed by the large transverse emittance and corresponds to about $1.1 \cdot 10^{-5}$ in time resolution. By correcting the chromaticity (red distribution in Fig. 5) the influence of the transverse motion can be significantly reduced and a resolution of up to $\Delta T / T \approx 2 \cdot 10^{-6}$ in a full momentum acceptance range can be achieved. Further improvements can be performed with octupole magnets (see the black distribution on the right picture in Fig. 5). In that case a resolution of up to $\Delta T / T \approx 3 \cdot 10^{-7}$ can be reached. Recently, all simulations were performed not taking into account the magnets' imperfections, which negatively act on the time resolution. Their influence can be crucial and will be studied and possibly corrected.

\section{References}

[1] FAIR Baseline Technical Report, GSI, Darmstadt, 2006, [http://www.gsi.de/fair]

[2] R. Maier, The High-Energy Storage Ring (HESR), Proceedings of PAC Conference, 2104-2106 (2011).

[3] A. Vasiliev et al., contribution to this conference.

[4] A. Dolinskii, et al., Lattice consideration for the Collector and the Accumulator ring of the FAIR project, Proceedings of COOL Conference, 106-109 (2007).

[5] U. Laier, et al., Design of an MA based RF system for the Collector Ring (CR), Proceedings of PAC Conference, 867-869 (2009).

[6] F. Nolden et al., Stochastic cooling in the framework of the FAIR project at GSI, Proceedings of EPAC Conference, 3479-3481 (2008).

[7] C. Peschke, F. Nolden, Pick-up electrode system for the CR stochastic cooling system, Proceedings of COOL Conference, 194-197 (2007).

[8] H. Geissel et. al., The Super-FRS project at GSI, NIM B204, 71-85 (2003).

[9] M. Hausmann et. al., First isochronous mass spectrometry at the experimental storage ring ESR, NIM A 446, 569-580 (2000). 
[10] A. Dolinskii et al., Design of a collector ring for antiproton and rare isotope beams, Proceedings of EPAC Conference, 572-574 (2002).

[11] A. Dolinskii et al., Nonlinear effects studies for a large acceptance Collector Ring, Proceedings of EPAC Conference, 1177-1179 (2004).

[12] S. Litvinov et al., The isochronous mode for mass measurements in the planned CR storage ring, Proceedings of STORI Conference, 320-323 (2005).

[13] S. Litvinov et al., The isochronous mode of the Collector Ring, Proceedings of EPAC Conference, 1618-1620 (2006).

[14] A. Dolinskii et al., Study of the mass resolving power in the CR storage ring operated as a TOF spectrometer, NIM A 574, 207-212 (2007). 